\title{
Integrin $\alpha v$ is necessary for the attachment of trophectoderm cells to osteopontin to mediate adhesion of the conceptus to the uterus during implantation in pigs
}

\author{
James W. Frank ${ }^{1}$, Fuller W. Bazer ${ }^{3}$, Robert C. Burghardt', Kayla J. Bayless² \\ and Gregory A. Johnson ${ }^{1}$ \\ ${ }^{1}$ Department of Veterinary Integrative Biosciences, Texas A\&M University; ${ }^{2}$ Department of Molecular \\ and Cellular Medicine, Texas A\&M Health Science Center; ${ }^{3}$ Department of Animal Science, Texas A\&M \\ University, College Station, Texas 77843 US
}

Pigs experience a significant loss of conceptuses during the attachment phase of implantation. The molecules that facilitate uterine-conceptus interactions in pigs are not fully understood, and this gap in knowledge limits our ability to improve reproductive efficiency. Osteopontin (OPN, also known as Secreted Phosphoprotein 1 or SPP1) is an emerging candidate marker for uterine receptivity to implantation in pigs. Expression of OPN by the uterus increases markedly during the peri-implantation period of pigs, as well as in other mammalian species, including sheep, goats, rabbits, mice and humans (Johnson '03, Johnson ‘09). In pigs, OPN mRNA is initially induced by conceptus estrogens in discrete regions of the uterine luminal epithelium (ULE) juxtaposed to the conceptus just prior to implantation on Day 13. OPN mRNA expands to the entire ULE by Day 20 when there is firm adhesion of conceptus trophectoderm to ULE (Garlow '02, White '05). OPN is a secreted extracellular matrix (ECM) protein that binds to cell surface integrins. Integrins are transmembrane glycoprotein receptors composed of non-covalently bound $\alpha$ and $\beta$ subunits that promote cell-cell and cell-ECM adhesion, cause cytoskeletal reorganization to stabilize adhesion, and transduce signals through numerous signaling intermediates. OPN potentially binds multiple integrins including $\alpha \mathbf{v} \beta 3, \alpha v \beta 1$, $\alpha v b 5, \alpha v b 6, \alpha 4 \beta 1, \alpha 9 \beta 1$ and $\alpha 8 \beta 1$. Our central hypothesis is that OPN secreted by the ULE binds integrins expressed by both ULE and trophectoderm to mediate implantation of the conceptus.

In a previous study, we tested whether $\alpha \mathrm{v}, \alpha 4, \alpha 5, \beta 1, \beta 3, \beta 5$, and $\beta 6$ integrin subunits expressed by porcine trophectoderm cells (pTr2) and ULE cells bound OPN. OPN bound the $\alpha v \beta 6$ integrin heterodimer on pTr2 cells and $\alpha v \beta 3$ on ULE cells (Erikson '09). Further, OPN promoted dose- and integrin-dependent attachment of pTr2 and ULE cells, and stimulated haptotactic pTr2 cell migration, meaning that cells migrated directionally along a physical gradient of non-soluble OPN. Finally, OPNcoated microspheres revealed co-localization of the $\alpha v$ integrin subunit and talin to focal adhesions at the apical domain of pTr2 cells.

The objectives of the current studies were to: 1$)$ determine mRNA expression and hormonal regulation of $\alpha v, \beta 3$, and $\beta 6$ integrins in the uterus and placenta; 2) determine the temporal and spatial assembly of $\alpha v-, \beta 3-$, and $\beta 6$-containing focal adhesions at the uterine-placental interface; and 3) determine whether integrin $\alpha v$ knockdown results in decreased attachment of pTr cells to OPN in vitro. In Experiment 1, gilts were hysterectomized on Day 9, 12 or 15 of the estrous cycle, or Day $9,12,15,20,25,30,35,40,50,60$ or 85 of pregnancy and uterine/placental tissues were subjected to in situ hybridization and immunofluorescence (IF) analyses to detect integrin subunit $\alpha v, \beta 3$, and $\beta 6$ mRNA and $\alpha v$ and $\beta 3$ protein at the maternal-conceptus interface. In Experiment 2, cyclic gilts were injected daily (Days 11-14) with 17-beta-estradiol benzoate (i.m.) or vehicle, and hysterectomized on Day 15 of pseudopregnancy. In Experiment 3, gilts were ovariectomized on Day 12 , injected daily with progesterone (i.m.) or vehicle for 28 days, and hysterectomized on Day 40. For Experiments 2 and 3, uteri were subjected to in situ hybridization analyses to determine

E-mail: JFrank@cvm.tamu.edu 
whether expression levels of $\alpha \mathrm{v}, \beta 3$ and $\beta 6 \mathrm{mRNAs}$ are regulated by estrogen and/or progesterone. In Experiment 4, custom smart pools of small interfering RNA (siRNA) targeted to GAPDH or pig $\alpha \mathrm{V}$ integrin were delivered to $\mathrm{pTr} 2$ cells to silence GAPDH or $\alpha \mathrm{v}$ gene expression respectively. Knock down of integrin subunit $\alpha \mathrm{v}$ in siRNA treated pTr2 cells was confirmed by Western blotting and IF analyses. pTr2 cells were then seeded evenly in 96-well plates in which wells were coated with either BSA (negative control), bovine fibronectin (FN), OPN, or type I collagen (positive control). The cells were allowed to adhere for $1 \mathrm{~h}$, stained with amido black and absorbance readings were taken at a wavelength of $595 \mathrm{~nm}$. Treated cells were also placed on OPN coated cover slips and IF was performed to visualize the $\alpha$ v integrin subunit.

Interestingly in situ hybridization analysis demonstrated that $\alpha \mathrm{v}, \beta 3$, and $\beta 6$ mRNAs increased in ULE and trophectoderm cells on Day 15 of pregnancy, peaked at Day 25, then decreased to background levels by Day 30 of gestation. No appreciable amounts of $\alpha \mathrm{v}, \beta 3$, or $\beta 6 \mathrm{mRNAs}$ were present in ULE of Day 15 pseudopregnant or Day 40 progesterone-treated gilts. IF staining detected large aggregates of $\alpha \mathrm{v}$ protein at the apical surfaces of ULE and trophectoderm cells, and $\beta 3$ aggregates on the ULE on Day 25 of pregnancy. Unexpectedly, these integrin aggregates, suggestive of focal adhesion assembly, were no longer detectable by Day 50. Finally, knocking down $\alpha v$ integrin decreased the adhesion of pTr2 cells to OPN and FN, but did not affect adhesion to type 1 collagen which does not bind $\alpha \mathrm{v}$ (Figure 1A).

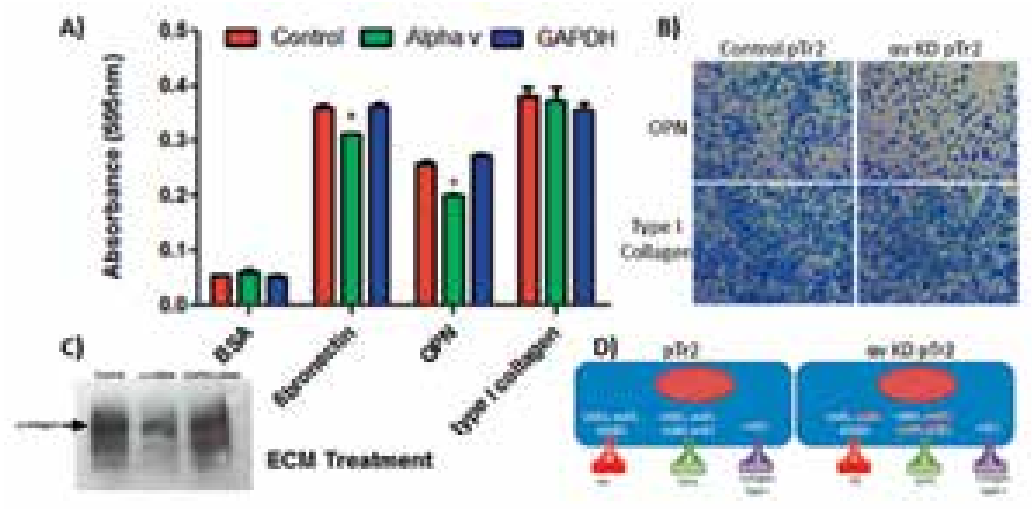

Fig. 1A. Adhesion of $\mathrm{pTr} 2$ cells to fibronectin (FN), OPN, or type I collagen. B. Photographs of pTr2 cells (control or $\alpha v$ knockdown (KD) attached to OPN or type I collagen. C. Western blot showing significant KD of $\alpha v$ integrin subunit. D. Cartoon of integrin expression in wild-type $p$ Tr2 cells (left panel) and pTr2 cells treated with siRNA directed to the $\alpha v$ integrin subunit (right panel).

Novel results from the current study in pigs are: 1) large focal adhesions form to adhere ULE and placental trophectoderm only during the peri-implantation period; 2) integrin mRNA increases in LE and trophectoderm during the peri-implantation period, and the up-regulation is not the result of estrogen or progesterone, suggesting that focal adhesion formation may lead to outside-in integrin signaling to increase integrin transcription in a positive feedback loop; and 3) $\alpha v$ integrin is necessary for the attachment of trophectoderm cells to OPN in vitro, suggesting a similar during implantation in pigs.

\section{References}

Erikson DW, Burghardt RC, Bayless KJ ' \& Johnson GA ${ }^{\mathbf{t}} 2009$ Secreted phosphoprotein 1 (SPP1, osteopontin) binds to integrin alphavbeta6 on porcine trophectoderm cells and integrin alphavbeta3 on uterine luminal epithelial cells, and promotes trophectoderm cell adhesion and migration. Biol Reprod 81 814-825.

Garlow JEt, Ka H', Johnson GA, Burghardt RC, Jaeger LA \& Bazer FW 2002 Analysis of osteopontin at the maternalplacental interface in pigs. Biol Reprod 66 718-725.

Johnson GA, Bazer FW, Burghardt RC, Spencer TE, Wu G \& Bayless KJ 2009 Conceptus-uterus interactions in pigs: endometrial gene expression in response to estrogens and interferons from conceptuses. Soc Reprod Fertil Suppl 66 321-332.

Johnson GA, Burghardt RC, Bazer FW \& Spencer TE 2003 Minireview: Osteopontin: roles in implantation and placentation. Biol Reprod 69 1458-1471.

White FJ, Ross JW, Joyce MM, Geisert RD, Burghardt RC \& Johnson GA 2005 Steroid regulation of cell specific secreted phosphoprotein 1 (osteopontin) expression in the pregnant porcine uterus. Biol Reprod 73 1294-1301. 